\title{
Performance Evaluation of ACO based Metaheuristic Technique for Color Image Segmentation
}

\author{
Baljot Kaur \\ M.Tech Scholar, \\ Department of Computer \\ Science \& Engineering, \\ Jalandhar, Punjab
}

\author{
P. S. Mann \\ Assistant Professor, \\ Department of Computer \\ Science \& Engineering, \\ Jalandhar, Punjab
}

\begin{abstract}
The aim of image segmentation is to make simpler presentation of an image into incredible which is meaningful as well as easy to understand. It is mainly utilized to know the location of objects, boundaries, lines etc in the digital images. Clustering technique is a method which shows the data set or pixels are replaced by cluster, pixels might be together because of the same color, texture etc. This paper represents the implementation of an ACO based metaheuristic for color image segmentation to differentiate the mixed regions.
\end{abstract}

\section{Keywords}

Image Processing, Image Segmentation Techniques, FELICM, Ant Colony Optimization.

\section{INTRODUCTION}

Image segmentation is the process of partitioning a digital image into multiple segments (sets of pixels, also known as super pixels). The goal of segmentation would be to simplify and/or change the representation of an image into something that's more meaningful and more straightforward to analyze. It forms a crucial preliminary step for subsequent object recognition and interpretation. Its goal would be to partition confirmed image into regions that have distinct objects. The most common kind of segmentation is on the basis of the assumption that distinct objects in an image have different and approximately constant colors.
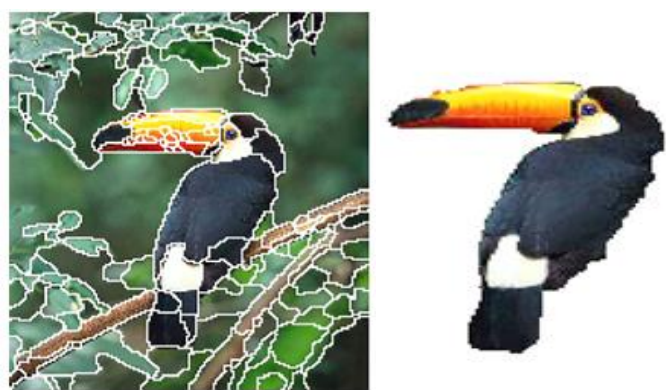

Fig: 1 (a)Mean Shift Segmentation (b) Segmentation Result

\section{IMAGE SEGMENTATION TECHNIQUES}

Based on different technologies, image segmentation approaches are currently divided into following categories, based on two properties of image.

\subsection{Detecting Discontinuities}

It means to partition an image based on abrupt changes in intensity [1], this includes image segmentation algorithms like edge detection.

\subsection{Detecting Similarities}

It means to partition an image into regions that are similar according to a set of predefined criterion [1]; this includes image segmentation algorithms like thresholding, region growing, region splitting and merging.

\section{FELICM}

Fuzzy C-Means that advantage and Local data is recognized as FELICM, it presents the pixels' weights inside local neighbor windows to reduce the edge degradation. This type of process can be utilized to conquer the isolated allocation of pixels with in sections of image.

\subsection{Mean Shift}

It's a way of clustering segmentation which is a non parametric iterative algorithm. It's applied during kernel thickness estimation. For every data point, mean transfer shows a window approximately as well as computes the mean of data point.

\section{ANT COLONY OPTIMIZATION}

This ACO algorithm is depending on meta-heuristic looking algorithm. This reflection is scrounging actions from ant varieties motivated and ant colony optimization approach improvement of random direct seek approach. Example, ants discover nearest course relating to the ant colony as well as a meals source by means of swapping specifics of your option that you should adopted. If the ants go through grub origin, they give secretions and incapacitated; such secretion chase can be applied near ants for convey collectively. Ant's probability would choose to adopt some sort of way relative to the amount from secretion. Applying straightforward responsive agencies permits the conversion through the natural for unreal auld like settlement. These factors interact personally through swapping data by means of surrounding alterations. It can be unreal pismire speak circuitously by means of unreal secretion chase. This offered near works on the particular list of ants acting forward to a picture determined through the local picture strength valuations edition. This kind of variance ensures a secretion matrix, while using like sizing picture, while in turn acts the particular boundary data and every picture place. Referable for big graphic sizing in order to cut calculation clip, the particular ant colony optimization approach depicted under was severally used upon non overlapping $128 * 128$ graphic windows. Ant Colony Optimization is a loop approach. 


\section{RELATED WORK}

Islam, Mohammed et al. [1] developed a method utilizing edge-based image segmentation methods forquality inspection that satisfies the professional requirements in pharmaceutical applications. The evaluation of an image with this might require complicated image processing techniques. Artan, Yusuf et al. [2] introduced a semi-supervised image segmentation method using machine learning techniques and super pixels. The proposed method yielded superior segmentation results over several semisupervised methods including the popular random walker algorithm. Huilin, Gao et al. [3] presented a technique of CT image segmentation based on region growing method, and the application form of effective segmentation of the lung tissue to chest CT images. Weighed against any other segmentation methods, the outcome showed that the segmentation method that has been proposed in this paper does better in CT image segmentation. The outcomes obtained have the significance in clinical applications and auxiliary diagnosis. Ji, Hongwei [11] presented an auto-context model (ACM)-based automatic liver segmentation algorithm, which combined ACM, multiatlases and mean-shift techniques to segment liver from 3-D CT images. The proposed method evaluated on the datasets of MICCA liver segmentation challenge. Mohan, K. Raj, and G. Thirugnanam [12] proposed a dualistic sub-image histogram equalization based enhancement and segmentation techniques. The proposed method has been tested and evaluated on several medical images. Jabar, Farah HA et al. [13] aimed to segment the blood cell images of patients experiencing acute leukemia having an adaptive K-Means clustering along with mean shift algorithm. The integrated clustering techniques have produced comprehensive output images with minimal filtering process to remove the background scene.

\section{GAPS IN LITERATURE}

These are the gaps in existing research on image enhancement methods.

1. The effect of mixed regions on image segmentation has ignored in the most of the existing techniques.

2. The color images contains maximum information for efficient image segmentation but majority of researchers has ignored it during image segmentation

3. The use of the ant colony based edge detector has also ignored in the majority of existing research on image segmentation.

\section{METHODOLOGY AND RESULTS \\ 7.1. Methodology}

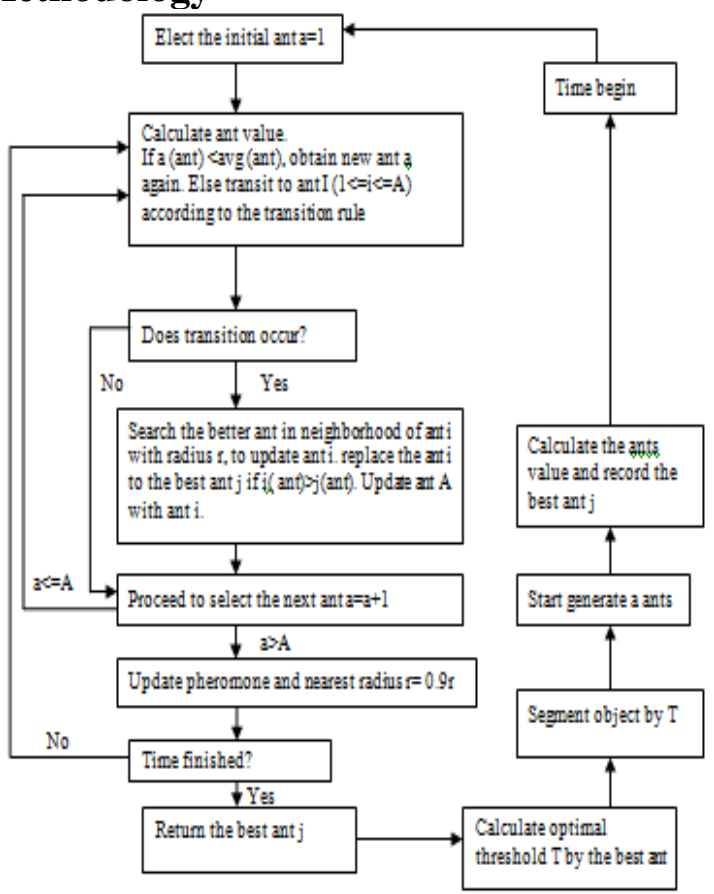

Fig.2. Methodology used for purposed algorithm

\subsection{Experimentation and results}

For experimentation and implementation the proposed technique is evaluated using MATLAB tool u2013a. Here we will compare the Gray Stretch Based algorithm for image segmentation and Principal Component Analysis based gray stretch algorithm on the basis of various image quality evaluation parameters like BER. The existing methodology give good results in enhancing the image but it neglects certain regions in image such as mixed region and also effect of color is not taken into consideration. The proposed approach gives efficient results in improving the contrast of image and it uses Principal Component Analysis based on Grey Stretch Algorithm. The tabular and graphical comparison has been done between existing and proposed methodology on the basis of parameters like accuracy, normalized cross correlation, structural similarity index metric and bit error rate.

The segmented images with proposed method:-

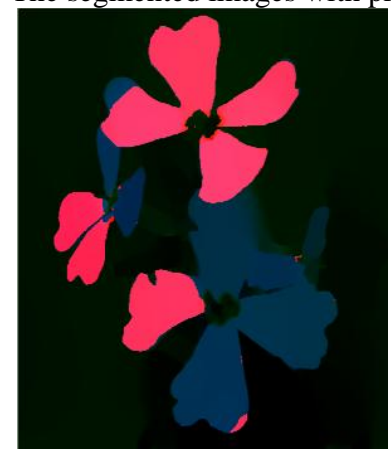

Fig 3: a) Image 1

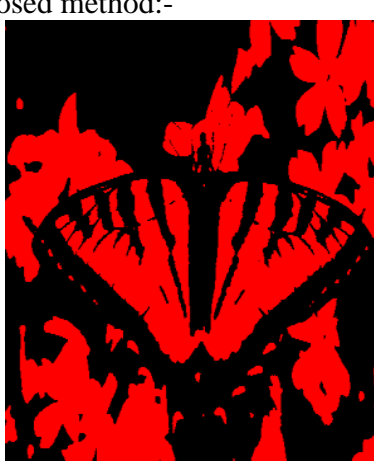

b) Image 2

\subsubsection{Accuracy}

Accuracy for image 1 in proposed method is 0.7028 and 0.9791 for image 2 . 


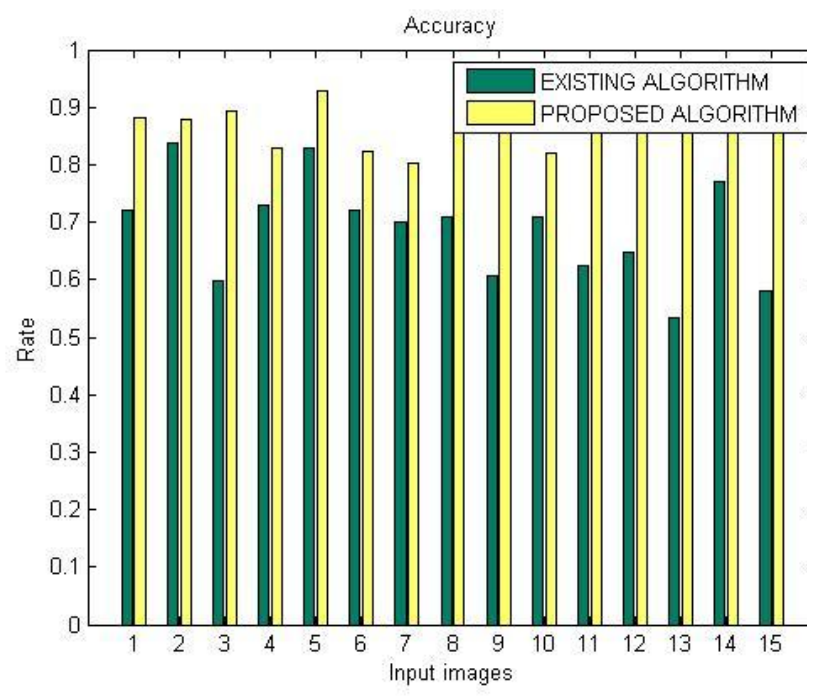

Fig 4: Accuracy

Table 1 has shown the quantized analysis of the accuracy. The proposed algorithm is higher as compared to existing method.

Table 1. Accuracy

\begin{tabular}{|c|c|c|}
\hline Image & Existing Results & Proposed Results \\
\hline 1 & 0.7222 & 0.8828 \\
\hline 2 & 0.8389 & 0.7483 \\
\hline 3 & 0.5971 & 0.8922 \\
\hline 4 & 0.7300 & 0.8293 \\
\hline 5 & 0.8297 & 0.9296 \\
\hline 6 & 0.7220 & 0.7028 \\
\hline 7 & 0.7000 & 0.8023 \\
\hline 8 & 0.7105 & 0.9336 \\
\hline 9 & 0.6077 & 0.8985 \\
\hline 10 & 0.7092 & 0.8203 \\
\hline 11 & 0.6245 & 0.9791 \\
\hline 12 & 0.6471 & 0.9062 \\
\hline 13 & 0.5343 & 0.8741 \\
\hline 14 & 0.7710 & 0.9211 \\
\hline 15 & 0.5799 & 0.9135 \\
\hline
\end{tabular}

\subsubsection{Normalized Cross Correlation-}

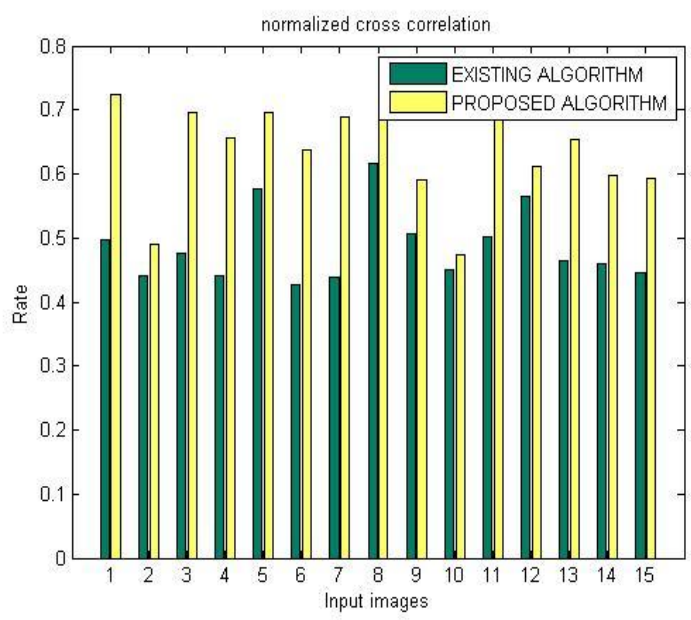

Fig 5: Normalized Cross Correlation

Table 2 has shown the quantized analysis of the normalized cross correlation. The proposed algorithm is higher as compared to existing method.

Table 2. Normalized Cross Correlation

\begin{tabular}{|c|c|c|}
\hline Image & Existing Results & Proposed Results \\
\hline 1 & 0.4962 & 0.7245 \\
\hline 2 & 0.4401 & 0.4895 \\
\hline 3 & 0.4750 & 0.6951 \\
\hline 4 & 0.4415 & 0.6563 \\
\hline 5 & 0.5771 & 0.6955 \\
\hline 6 & 0.4276 & 0.6382 \\
\hline 7 & 0.4384 & 0.6889 \\
\hline 8 & 0.6163 & 0.7040 \\
\hline 9 & 0.5075 & 0.5906 \\
\hline 10 & 0.4514 & 0.4736 \\
\hline 11 & 0.5022 & 0.7108 \\
\hline 12 & 0.5643 & 0.6112 \\
\hline 13 & 0.4643 & 0.6544 \\
\hline 14 & 0.4586 & 0.5976 \\
\hline 15 & 0.4459 & 0.5924 \\
\hline
\end{tabular}




\subsubsection{Structural Similarity Index Metric-}

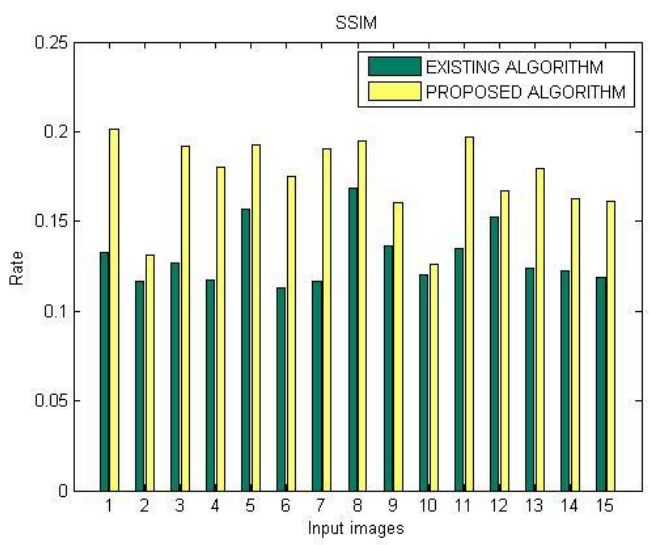

Fig 6: Structural Similarity Index Metric

Table 3 has shown the quantized analysis of the SSIM. The proposed algorithm is higher as compared to existing method.

Table 3. Structural Similarity Index Metric

\begin{tabular}{|c|c|c|}
\hline Image & Existing Results & Proposed Results \\
\hline 1 & 0.1329 & 0.2014 \\
\hline 2 & 0.1169 & 0.1309 \\
\hline 3 & 0.1268 & 0.1922 \\
\hline 4 & 0.1173 & 0.1803 \\
\hline 5 & 0.1565 & 0.1924 \\
\hline 6 & 0.1133 & 0.1748 \\
\hline 7 & 0.1164 & 0.1903 \\
\hline 8 & 0.1682 & 0.1950 \\
\hline 9 & 0.1361 & 0.1605 \\
\hline 10 & 0.1201 & 0.1264 \\
\hline 11 & 0.1346 & 0.1971 \\
\hline 12 & 0.1527 & 0.1667 \\
\hline 13 & 0.1237 & 0.1797 \\
\hline 14 & 0.1221 & 0.1626 \\
\hline 15 & 0.1185 & 0.1611 \\
\hline
\end{tabular}

\subsubsection{Bit Error Rate-}

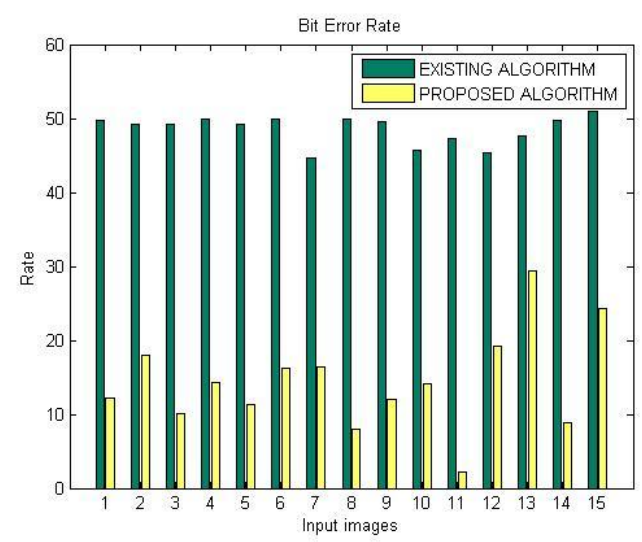

Fig 7: Bit Error Rate
Table 4 has shown the quantized analysis of the bit error rate. The proposed algorithm is higher as compared to existing method.

Table 4. Bit Error Rate

\begin{tabular}{|c|c|c|}
\hline Image & Existing Results & Proposed Results \\
\hline 1 & 49.7894 & 12.2045 \\
\hline 2 & 49.7365 & 18.0289 \\
\hline 3 & 49.2240 & 10.0813 \\
\hline 4 & 49.8897 & 14.2976 \\
\hline 5 & 49.2089 & 11.3493 \\
\hline 6 & 49.9795 & 16.3109 \\
\hline 7 & 44.6594 & 16.3566 \\
\hline 8 & 49.9135 & 8.0244 \\
\hline 9 & 49.6193 & 11.9707 \\
\hline 10 & 45.6523 & 14.0958 \\
\hline 11 & 47.2365 & 2.2476 \\
\hline 12 & 45.3514 & 19.1879 \\
\hline 13 & 47.6506 & 29.4595 \\
\hline 14 & 49.8232 & 8.8461 \\
\hline 15 & 50.9227 & 24.2136 \\
\hline
\end{tabular}

\section{CONCLUSION}

This paper represents the performance of ant colony based edge detector to differentiate the mixed regions i.e. proposed technique as well shows the Fuzzy C-Means with Edge and Local Information (FELICM), existing technique which introduces the pixels' weights within local neighbor windows to decrease the edge degradation. This approach has somehow used to overcome the isolated distribution of pixels inside segments of image and the comparison between the techniques i.e. proposed results has shown significant improvement. The implemented an ACO based metaheuristic for color image segmentation is done using parameters like accuracy, normalized cross correlation, structural similarity index metric and bit error rate. In the near future more evolutionary optimizations techniques will be used for color images for further enhancement.

\section{REFERENCES}

[1] Islam, Mohammed J., Saleh Basalamah, Majid Ahmadi, and Maher A. Sid-Ahmed. "Capsule image segmentation in pharmaceutical applications using edge-based techniques." In Electro/Information Technology (EIT), 2011 IEEE International Conference on, pp. 1-5. IEEE 2011.

[2] Artan, Yusuf. "Interactive image segmentation using machine learning techniques." In Computer and Robot Vision (CRV), 2011 Canadian Conference on, pp. 264269. IEEE, 2011.

[3] Huilin, Gao, Dou Lihua, Chen Wenjie, and Xie Gang. "The applications of image segmentation techniques in medical CT images." In Control Conference (CCC), 2011 30th Chinese, pp. 3296-3299. IEEE, 2011.

[4] Zhu, Shaohua, and Zhaohua Wu. "Study on solder joint image segmentation techniques based on Matlab." In Electronic Packaging Technology and High Density 
Packaging (ICEPT-HDP), 2011 12th International Conference on, pp. 1-3. IEEE, 2011.

[5] Khanna, Anita, and Manish Shrivastava. "Unsupervised techniques of segmentation on texture images: A comparison." In Signal Processing, Computing and Control (ISPCC), 2012 IEEE International Conference on, pp. 1-6. IEEE, 2012

[6] Chebbout, Samira, and Hayet Farida Merouani. "Comparative Study of Clustering Based Colour Image Segmentation Techniques." In Signal Image Technology and Internet Based Systems (SITIS), 2012 Eighth International Conference on, pp. 839-844. IEEE, 2012.

[7] Samet, R., S. E. Amrahov, and A. H. Ziroglu. "Fuzzy Rule-Based Image Segmentation technique for rock thin section images." In Image Processing Theory, Tools and Applications (IPTA), 2012 3rd International Conference on, pp. 402-406. IEEE, 2012.

[8] Vij, Sugandhi, Sandeep Sharma, and Chetan Marwaha "Performance evaluation of color image segmentation using $\mathrm{K}$ means clustering and watershed technique." In 2013 Fourth International Conference on Computing, Communications and Networking Technologies (ICCCNT), pp. 1-4. IEEE, 2013.

[9] Rincon-Montes, V., A. Vargas-Olivares, Samuel Pichardo, Laura Curiel, and J. E. Chong-Quero. "Quantitative evaluation method of image segmentation techniques for Magnetic Resonance guided High Intensity Focused Ultrasound therapy." In Electrical Engineering, Computing Science and Automatic Control (CCE), 2013 10th International Conference on, pp. 110-115. IEEE, 2013.

[10] Jiang, Ching-Fen, and Ka-Pei Tsai. "Image segmentation techniques for stem cell tracking." In Acoustics, Speech and Signal Processing (ICASSP), 2013 IEEE International Conference on, pp. 1109-1112. IEEE, 2013.

[11] Ji, Hongwei, Jiangping He, Xin Yang, Rudi Deklerck, and Jan Cornelis. "ACM-based automatic liver segmentation from 3-D CT images by combining multiple atlases and improved mean-shift techniques." IEEE journal of biomedical and health informatics 17, no. 3 (2013): 690698.
[12] Mohan, K. Raj, and G. Thirugnanam. "A dualistic subimage histogram equalization based enhancement and segmentation techniques for medical images." In Image Information Processing (ICIIP), 2013 IEEE Second International Conference on, pp. 566-569. IEEE, 2013.

[13] Jabar, Farah HA, Waidah Ismail, Rosalina Abdul Salam, and Rosaline Hassan. "Image Segmentation Using an Adaptive Clustering Technique for the Detection of Acute Leukemia Blood Cells Images." In Advanced Computer Science Applications and Technologies (ACSAT), 2013 International Conference on, pp. 373-378. IEEE, 2013.

[14] Weingart, Mircea, and Orest Vascan. "Image segmentation processing-some techniques and experimental results A comparative study of the concepts of some segmentation techniques." In Electrical and Electronics Engineering (ISEEE), 2013 4th International Symposium on, pp. 1-6. IEEE, 2013.

[15] Li, Nan, Hong Huo, Yu-ming Zhao, Xi Chen, and Tao Fang. "A spatial clustering method with edge weighting for image segmentation." IEEE Geoscience and Remote Sensing Letters 10 (2013): 1124-1128.

[16] Gandhi, Nupur J., Vandana J. Shah, and Ravindra Kshirsagar. "Mean shift technique for image segmentation and Modified Canny Edge Detection Algorithm for circle detection." In Communications and Signal Processing (ICCSP), 2014 International Conference on, pp. 246-250 IEEE, 2014

[17] Saranya, R., Jackson Daniel, A. Abudhahir, and N. Chermakani. "Comparison of segmentation techniques for detection of defects in non-destructive testing images." In Electronics and Communication Systems (ICECS), 2014 International Conference on, pp. 1-6. IEEE, 2014.

[18] Krishnan, P. Hari, V. Karthickeyan, and P. Ramamoorthy. "A novel method for measurement of fetal volume from US images using segmentation techniques." In Green Computing Communication and Electrical Engineering (ICGCCEE), 2014 International Conference on, pp. 1-6. IEEE, 2014 